\title{
A biologically derived pectoral fin for yaw turn manoeuvres
}

\author{
Jonah R. Gottlieb ${ }^{a *}$, James L. Tangorra ${ }^{b}$, Christopher J. Esposito ${ }^{\mathrm{b}}$ and George V. Lauder ${ }^{\mathrm{c}}$ \\ ${ }^{a}$ Applied Physics Laboratory, Johns Hopkins University, Laurel, Maryland, USA; ${ }^{b}$ Drexel University, Philadelphia, Pennsylvania, USA; \\ ${ }^{c}$ Harvard University, Cambridge, Massachusetts, USA
}

(Received 16 December 2008; final version received 8 December 2009)

\begin{abstract}
A bio-robotic fin has been developed that models the pectoral fin of the bluegill sunfish as the fish turned to avoid an obstacle. This work involved biological studies of the sunfish fin, the development of kinematic models of the motions of the fin's rays, CFD based predictions of the 3D forces and flows created by the fin, and the implementation of simplified models of the fin's kinematics and mechanical properties in a physical model. The resulting robotic fin produced the forces and flows that drove the manoeuvre and had a sufficiently high number of degrees of freedom to create a variety of non-biologically derived motions. The results indicate that for robotic fins to produce a level of performance on par with biological fins, both the kinematics and the mechanical properties of the biological fin must be modelled well.
\end{abstract}

Keywords: unmanned underwater vehicles (UUVs); bio-robotic; pectoral fin; kinematics; manoeuvring

\section{Introduction}

Pectoral fins are used by fish and aquatic animals to execute a wide variety of swimming manoeuvres. They can be used as rudders to control pitch during high speed swimming and to enhance low speed manoeuvrability (Drucker and Jensen 1996; Westneat 1996; Wilga and Lauder 1999, 2000, 2001; Lauder and Drucker 2004) and as flapping, high degree of freedom (DOF) propulsors to propel and manoeuvre the animal at low speeds (Lauder and Drucker 2004). Because of this versatility, the pectoral fin has served as a source of inspiration for a range of bio-robotic devices (Kato and Furushima 1996; Anderson and Chhabra 2002; Fish et al. 2003; Tangorra et al. 2006, 2008; Palmisano et al. 2007; Gottlieb et al. 2008). These robotic devices have been developed as alternatives for propellers and traditional control surfaces for underwater vehicles and have been used as experimental tools for investigations of the hydrodynamics and control of fish swimming.

The pectoral fin of the bluegill sunfish (Lepomis machrochirus) is an example of a fin that is used as a flapping propulsor for both steady swimming and manoeuvring. This fin is a highly deformable and controllable surface, which can be made to flap, feather, and row, as well as to take on a variety of complex three-dimensional shapes (Drucker and Lauder 2001; Lauder et al. 2007; Tangorra et al. 2007). These paired fins are often used synchronously and made to repeat the same fin beat over and over to produce the cyclic forces required for steady swimming or hovering and then made to execute completely different kinematics that, within a single fin beat, produce a drastic reorientation of the fish body.

In this paper, we described the development of a biorobotic fin that has the controllability, DOF and mechanical properties required to produce the motions, forces and flows of the sunfish pectoral fin during a yaw turn manoeuvre. To do so, we followed the design methodology developed originally for the construction of a bio-robotic fin used to investigate the mechanisms of thrust production during steady swimming (Tangorra et al. 2007, 2008). In this process, the kinematics and hydrodynamics of the bluegill sunfish pectoral fins were studied as fish executed turns to avoid obstacles, digital models of fin motions were created, predictions of the forces and flows were made using computational fluid dynamics (CFD) analyses, and the robotic model was designed to capture the aspects of the fin's kinematics and structure deemed most critical for producing the forces that drove the manoeuvre.

\section{Sunfish body and pectoral fins during a yaw turn}

\subsection{Motions}

The kinematics of the pectoral fin was studied as a sunfish executed a turn manoeuvre to avoid an obstacle. The manoeuvre was stimulated by placing a rod to the front left of the fish as the fish swam steadily in a flow tank at a speed of 0.5 body lengths per second. At this swimming speed, the pectoral fins are largely responsible for propulsion with minimal contributions coming from the fish tail or other

\footnotetext{
*Corresponding author. Email: Jonah.Gottlieb@jhuapl.edu
} 

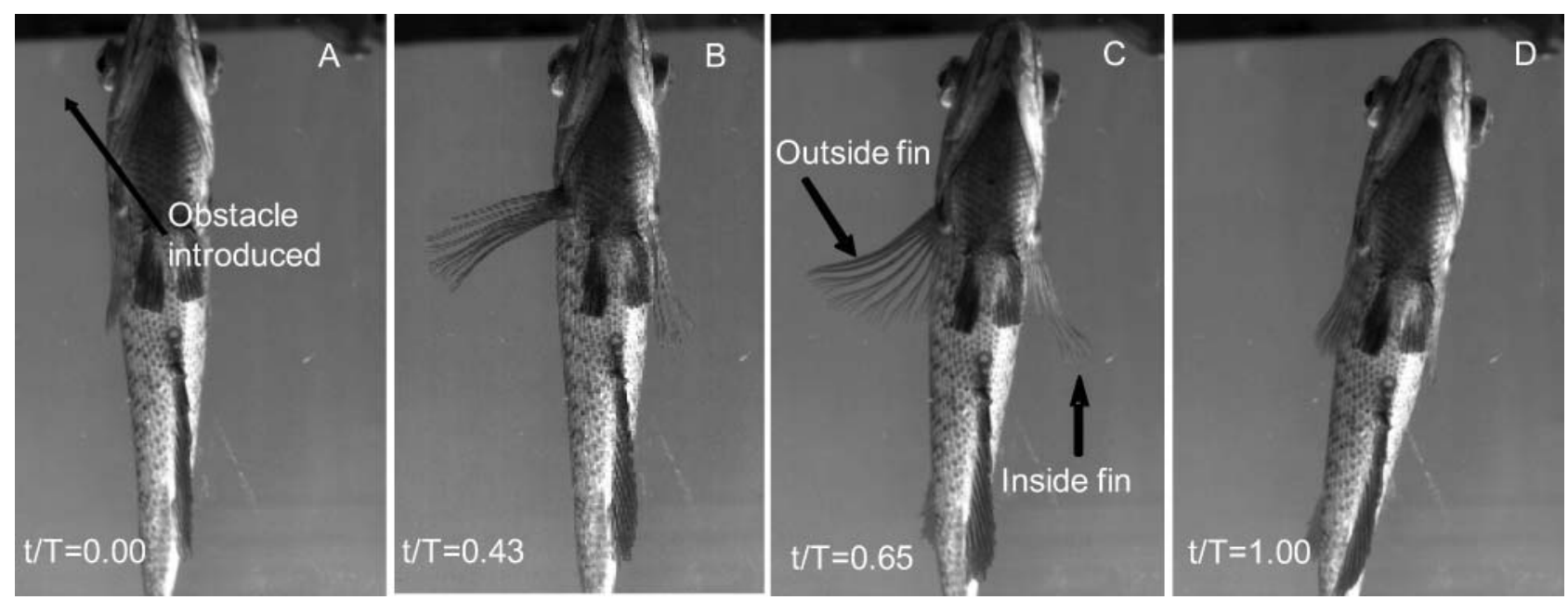

Figure 1. Ventral view of the sunfish performing a yaw turn. The introduction of an obstacle induces the fish to perform a yaw turn in order to avoid it. The pectoral fin on the outside of the turn is largely responsible for the motion of the fish.

fins (Drucker and Lauder 2001). To capture the body and fin movements, high speed, high resolution video was taken $(250 \mathrm{fps}, 1024 \times 1024)$ of the lateral and ventral views of the fish. The 3D coordinates of ten points along each of the fin's 14 fin rays were tracked through time and were used to create a digital model of fin ray movements through the turn manoeuvre.
The fish's response to the obstacle can be described as three overlapping actions: a large lateral movement away from the rod (Figure 1B, C), a slowing of the forward velocity (Figure 1C) and a slight downward motion (Figure 2C-F). The lateral movement, which consisted of both a rotation and a translation of the body away from its initial heading, occurred first. After the fish had begun
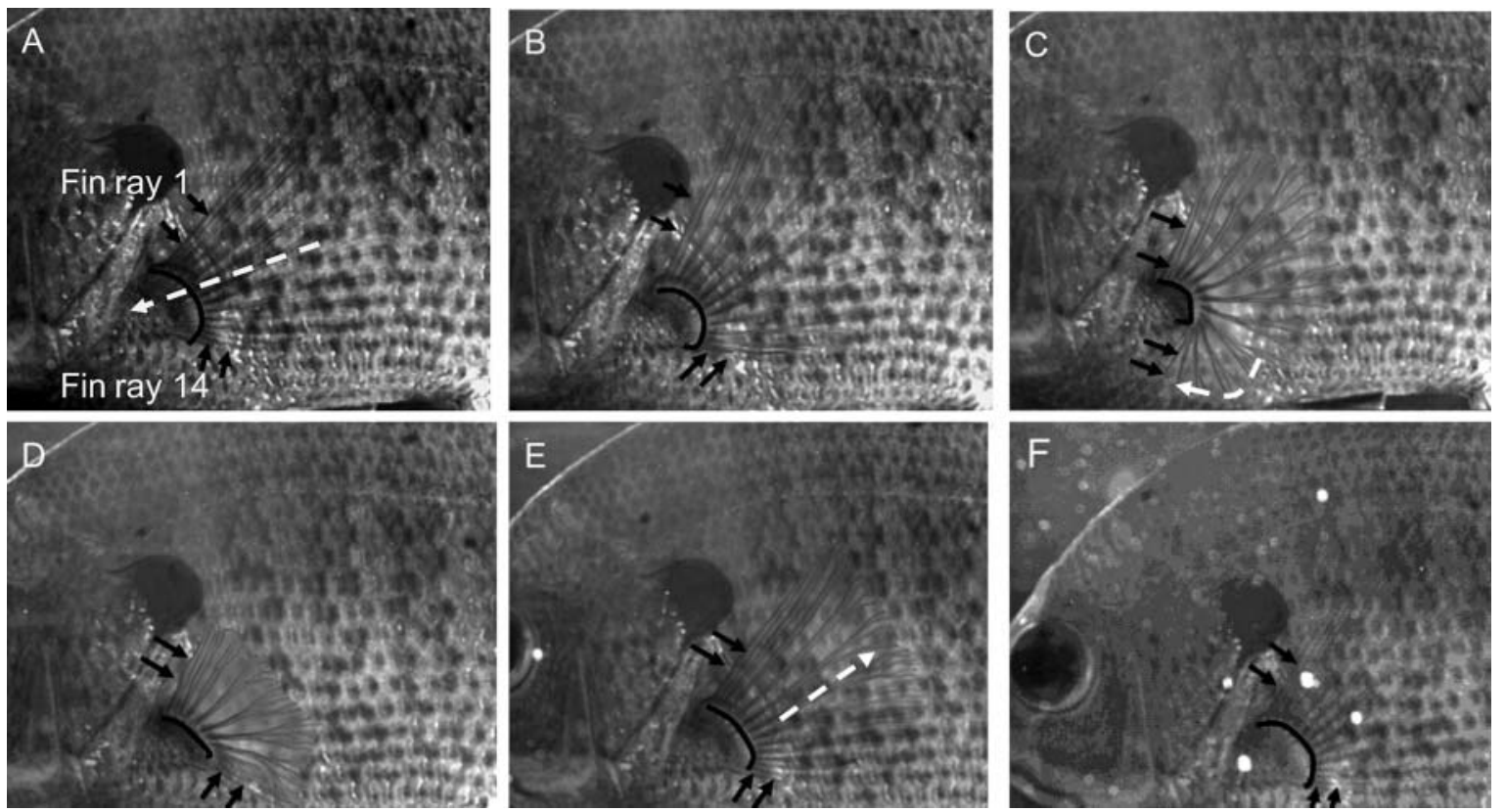

Figure 2. The pectoral fin on the outside of the turn. The fin starts against the body in frame A, sweeps through the outstroke (B, C), a transition period (D) and instroke (E) before returning to the body in frame F. Fin rays 1 and 14, the two leading edges are represented by the small, solid arrows. Dotted arrows show overall fin motions, and the curved solid line indicates the approximate shape of the fin base at each time. 
to move laterally away from the obstacle, it slowed down (which was also caused by the moving flow) and moved slightly downward away from the obstacle.

These motions were driven by the coordinated movements of the two pectoral fins, which performed very different motions from each other (Figure 1). The fin on the outside of the turn (right fin, strong side) had the more pronounced motion. During the outstroke, its upper and lower halves were led by the movements of the most dorsal and ventral fin rays (fin rays 1 and 14, respectfully), which moved $45^{\circ}$ to $60^{\circ}$ away from the fish body. During the instroke, the fin maintained a large area and moved strongly back towards the fish body. In comparison, the fin on the inside of the turn (left fin, weak side) moved much less. It extended through smaller angles $\left(30^{\circ}\right)$ and with significantly slower speeds during the outstroke. On the instroke, it seemed to maintain its absolute position in the water such that the fish body moved toward the fin rather than the fin being moved actively toward the fish body. From these observations, it was concluded that the fin on the outside of the turn drove the fish's movement and thus should serve as the focus for the bio-robotic fin. This decision was supported by the fact that the peak accelerations of the fish body were synchronous with the highest velocities of the outside fin. It was also apparent from an analysis of fin ray motions that a bio-robotic fin that was capable of creating the motions of the outside fin would also be able to generate the movements of the inside fin.

The complex motions of the strong side fin can be described largely by the motions of the fin's upper (dorsal) and lower (ventral) halves. The upper half is composed of fin rays 1 through 7, and the lower half, fin rays 8 through 14. By treating the two halves as independent elements and breaking the fin beat into the outstroke and instroke, fin motions of different types may be directly compared. The outstroke began with the forward sweep of the ventral half, which was led by fin ray 14 (Figure 2B, 2C and $\mathrm{Y} 2, \mathrm{Y} 3$ in Figure 3). A simultaneous downward rotation of the ventral half occurred during this initial sweep, creating a curvature in the fin's webbing. Next, the dorsal half of the fin swept forward. The ventral half then rotated upwards, and the two halves realigned such that the fin became approximately planar (Figure 2D and Y4 in Figure 3). The instroke followed this realignment (Figure 2E, 2F and Y5, Y6 in Figure 3). During the instroke, fin rays 1 and 14 led the middle rays on the fin's sweep back to the body.

These motions differ significantly from those made by the pectoral fin during steady swimming. During steady swimming, the left and right pectoral fins usually move in unison, and the motion of the fins is smoother and more graceful than during the impulsive yaw turn. As in the yaw turn, the motion during the outstroke is led by the movement of the most dorsal and ventral fin rays. The fin pulls away from the fish body and cups about its spanwise axis as it moves into the flow (S2, S3 in Figure 3). The dorsal

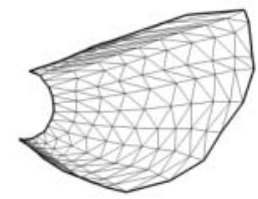

Y1
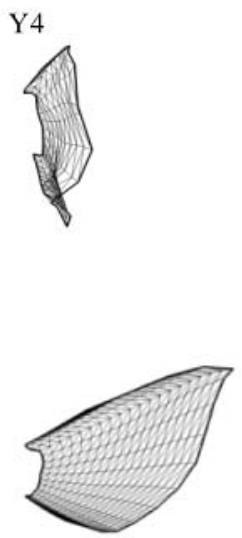

S1

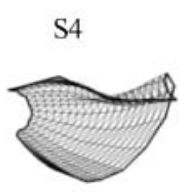

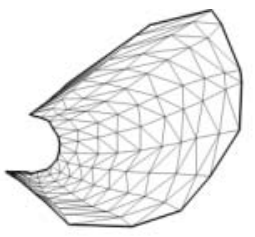

Y2

Y5

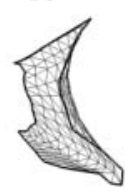

The Yaw Turn
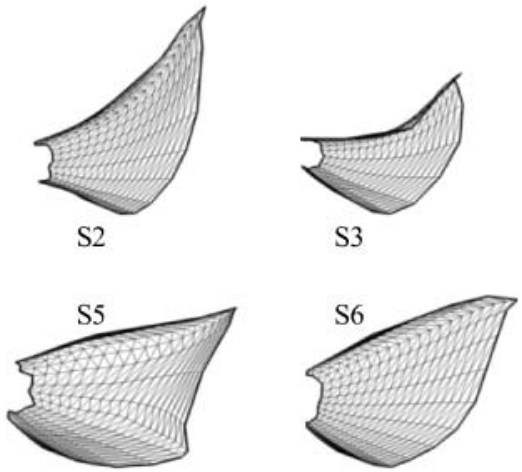

Steady Swimming

Figure 3. Comparison of fin motions during the yaw turn and a steady swimming stroke, lateral views. During the yaw turn, the movement of the strong side fin away from the body (Y1-Y4) is led by the ventral half of the fin. The fin is not pulled smoothly into the fish body during the instroke (Y5, Y6) but remains extended in the water (Y6) and returns to the body as the tail of the fish rotates towards the extended fin. During steady swimming, the fin cups about its spanwise axis as it is swept into the flow (S1-S4), with a wave of bending travelling along the upper edge (S3, S4).

half rotates forward and downward, and a wave of bending travels along the upper leading edge (S3, S4 in Figure 3). The dorsal edge sweeps forward about $60^{\circ}$; the ventral edge, approximately $45^{\circ}$. During the instroke, the fin uncups, it expands in area (S5 in Figure 3) and then returns to the body (S6 in Figure 3).

The stiffness of the fin is very important to the production of force, as it influences the dynamic interaction and exchange of energy between the fin and the water. As occurs with fin ray kinematics, there is strong evidence that the fish actively modulates the stiffness of the fin rays so that it is appropriate for the specific swimming gait and swimming speed (Tangorra et al. 2008). During steady swimming, the pectoral fin is very compliant; the tips of the fin rays bend and twist away from the direction of movement (S3, S4 in 
Figure 3). During the outstroke of the yaw turn, however, the fin appeared visually to be stiffer (Y3, Y4 in Figure 3). There is less bending of the distal end despite fin velocities being very similar to those during steady swimming. This apparent change in stiffness also had to be modelled properly for the robotic fin to produce biologically relevant results.

\subsection{Forces and flows}

The forces and flows created during the yaw turn manoeuvre were predicted numerically using computational fluid dynamics (CFD) (Ramakrishnan et al. 2008). Numerical models of the fin's motions were developed using the 3D coordinates of the fin rays through time. The timing and magnitudes of the forces and flows were consistent with what would be expected to produce the fish's movement (Figure 1). During the outstroke, the fin created a large contralateral force and a smaller drag force, which drove the fish away from the obstacle and slowed its movement. A small lift force was also produced during this time, which was an order of magnitude smaller than either the lateral or drag forces. During the instroke, ipsi-lateral, thrust and an even smaller downward force were created. Their peak magnitudes were smaller than what they were during the outstroke.

In general, the largest force created during the manoeuvre, the lateral force, was up to four times as large as the largest force developed during steady swimming, the thrust force. The forces created by the outside manoeuvring fin were an order of magnitude larger than those of the inside fin (Ramakrishnan et al. 2008).

The lateral force created during the outstroke was the result of the fin pushing a strong lateral jet of fluid away from the body. CFD identified a large vortex that formed near the upper tip of the fin, which continued to develop as the fin moved through the manoeuvring stroke. The vortex ring that developed during the manoeuvre, through which the lateral jet was formed, remained normal to the fish body throughout the stroke. This ensured that the largest force would remain in the lateral direction, moving the fish away from the obstacle. This strong lateral jet is consistent with the type of flows that would be responsible for driving the fish body away from the obstacle.

\section{Design of the bio-robotic fin}

\subsection{General design}

The design of this bio-robotic fin (Figure 4) was an evolution of a fin built originally to study the motions of the sunfish's pectoral fin during steady swimming (Tangorra et al. 2007, 2008). The fin is composed of five fin rays, each mounted on hinges (Robart Mfg. Inc, St. Charles IL, USA) that sit within a plastic base that serves as the pectoral fin girdle (Figure 5). The hinges allow the fin rays to be swept fore and aft, and three of the five fin rays sit in bases that can also be rotated laterally. The fin rays and base were constructed from ABS plastic using a fused deposition modelling machine (Dimension Elite, Stratasys, Inc., Eden Prairie, MN). The fin rays are scaled to be approximately four times the length of average sunfish fin rays such that the longest ray measures $160 \mathrm{~mm}$ from base to tip. The fin rays are covered in a webbing made using an $80 \% / 20 \%$ polyester/elastane blend. Servomotors (HSR-5990TG, Hitec RCD, Poway CA, US) actuate each fin ray by pulling on low stretch, polyethylene tendons attached to the fin ray bases (Gel spun 35-lb test, The Orvis Company, Inc., Manchester, VT). The motors are commanded via a servomotor control board (Lynxmotion SSC-32 Servo Control Board, Lynxmotion Inc., Pekin, IL, US), which executes a control program written in Visual Studio 2008 (Microsoft Corp., Redmond, WA, US). The result was a robotic fin with a sufficiently high number of actuated degrees of freedom to execute the fin motions used in a yaw turn, as well as those used in other swimming motions.

\subsection{Fin ray trajectories}

The trajectories through which the robotic fin rays were moved were based on 1- and 2-DOF models of the trajectories of the biological rays. These driven motions of the fin were determined by modelling the motions of the roots of the biological fin rays. Although the $3 \mathrm{D}$ positions along the full length of each fin ray throughout the stroke were collected, only the three points at the base of each ray were used to develop models of the driven motion. The distal end of the fin bends considerably due to hydrodynamic loading, but the root, which is significantly stiffer, remains rather straight throughout the fin beat. The motions of the fin ray roots, therefore, represent the driven motion of the fin rather than the dynamic motions of the full fin. Lines were fitted to three points at the bases of fin rays 1, 4, 7, 10 and 14 at evenly spaced time steps throughout the manoeuvre. These lines intersected each other within a small region, which was approximated as the centre of rotation for that fin ray. This approximation ignores any translation made by the fin rays. The simplest pathways, which were followed by fin rays 4 and 7 , could be modelled as a rotation in a plane about a single centre of rotation. The trajectories for the most ventral $(10,14)$ and most dorsal (1) fin rays were more complex and were modelled as elliptical rotations about a single point. These elliptical rotations were modelled decomposed into simultaneous rotations through two orthogonal planes and sharing a common rotation point: a sweep rotation in the fore-aft plane and a lateral rotation in the other. This 2-DOF modelling allowed the $3 \mathrm{D}$ rotations to be modelled in much the same way as fin rays 4 and 7 but with an addition plane of rotation, requiring a more complicated mechanical joint.

To drive the robotic fin rays along the complex trajectories associated with the yaw turn, it was required that each 

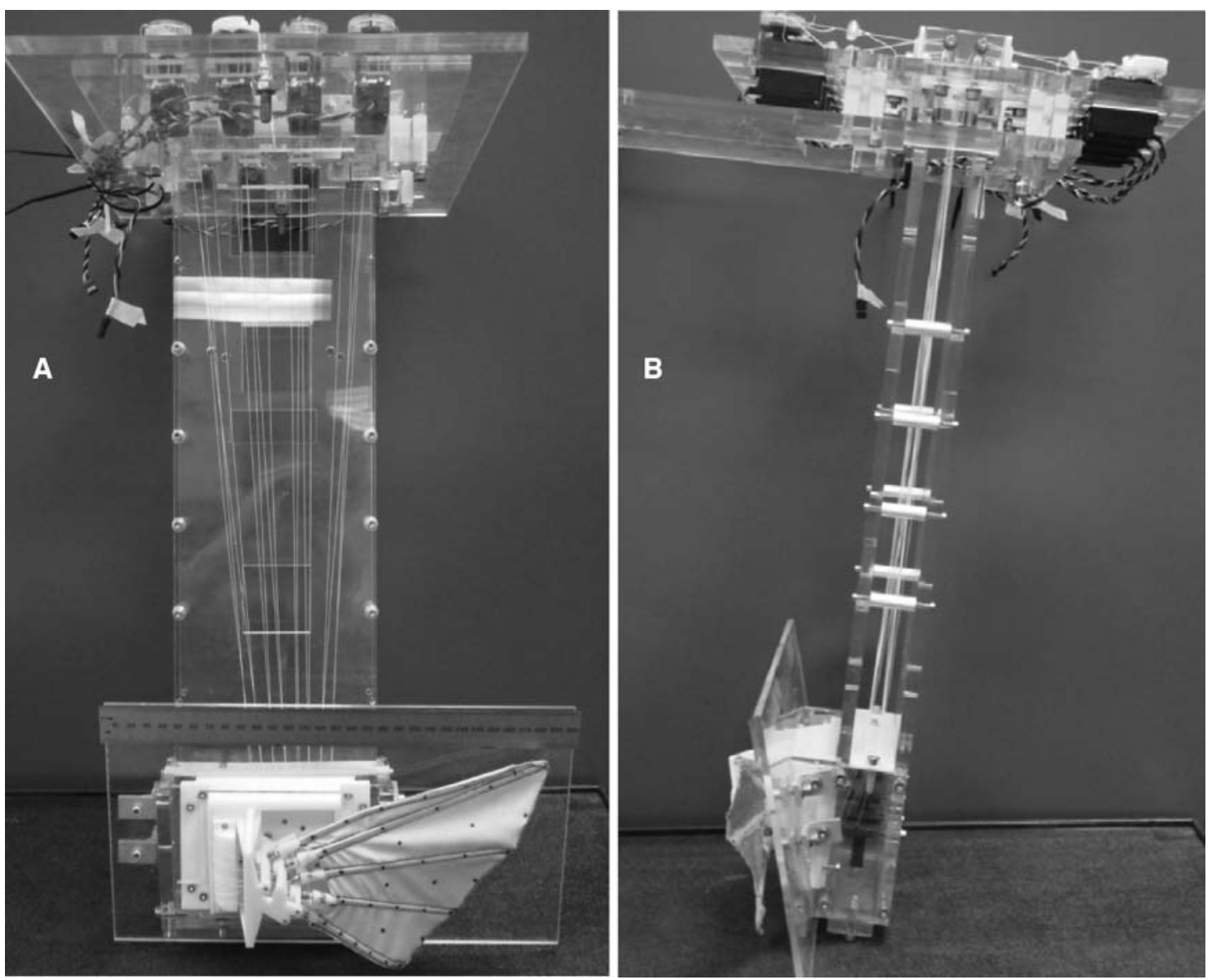

Figure 4. Front (A) and side (B) views of the fin and motor mount. Ruler in frame A is $300 \mathrm{~mm}$ long. The structure is approximately $300 \mathrm{~mm}$ wide at the bottom plate, meant to simulate the fish body, $600 \mathrm{~mm}$ tall and only $50 \mathrm{~mm}$ thick. The controlling tendons attach to the fin at the bottom, run the height of the structure and attach to the servo motors mounted above.

fin ray be actuated using a unique trajectory, some of which contained a second plane of rotation (Figure 6). The steady swimming fin required a less complicated control strategy, and all five fin rays were able to be driven using the same trajectory with the curvature of the base responsible for creating the proper fin shapes.

\subsection{Base design}

A base of rigid plastic was designed to support the fin rays and facilitate the rotational fin ray trajectories (Figure 5). The positions of the centres of rotation determined the shape and size of the base, which measures approximately $70 \mathrm{~mm}$ across (from dorsal most to ventral most rays).

Since the fin rays underwent very different motions for the manoeuvre and steady swimming strokes, their centres of rotation created different base curvatures in the two fins. The distance between the leading edge fin rays on this manoeuvring fin (Figure 7A) is much greater than it is on the steady swimming fin (Figure 7B) due largely to the pro- nounced role that the fin's ventral portion plays in the yaw turn. On the steady swimming fin, the placement of the leading edge fin rays is such that they are approximately in line with each other and slightly forward of the medial fin rays.

\subsection{Fin rays}

The bio-robotic fin rays required structural properties that would give them an appropriate dynamic interaction with the water and direct forces along the same 3D paths as the biological fin rays. This was accomplished by tapering fin ray cross sections so that the robotic fin rays had flexural rigidities along their length that were scaled proportionally to those of the biological fin rays (Alben et al. 2006; Tangorra et al. 2008). Using this modelling technique, the manoeuvring fin was initially given fin rays with flexural rigidities that were $2000 \times$ those of the biological fin rays. This value was twice of that used in the stiffer steady swimming fins and was based on modelling developed for the steady 


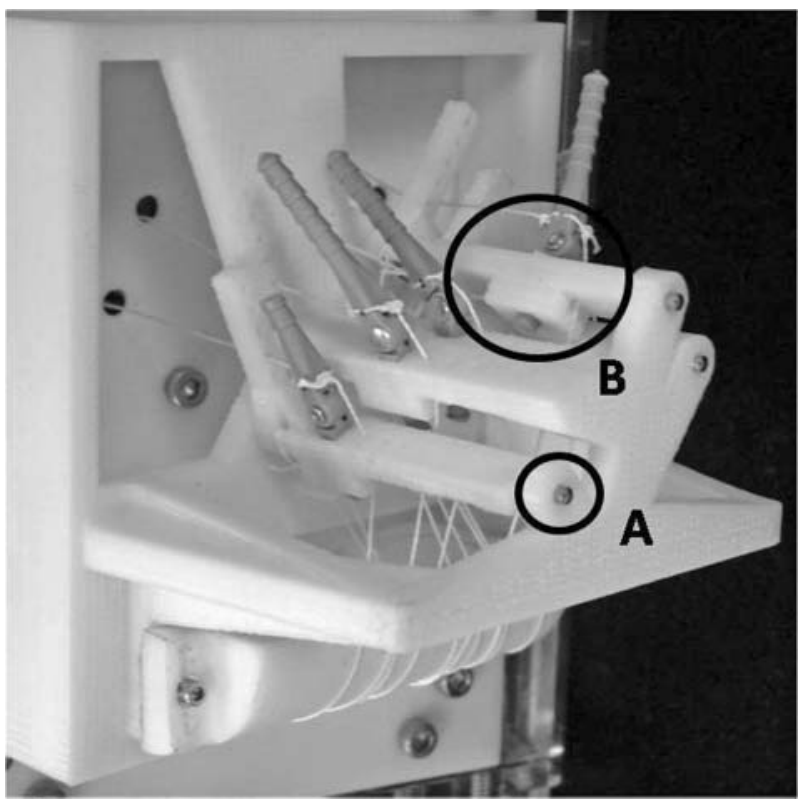

Figure 5. Base close-up. Visible are metal pins used as pivots (detail A) for 2-DOF joints (detail B). Actuating tendons attach to grey hinges on which the fin rays (not shown) are mounted.

swimming fins (Tangorra et al. 2008). The value was justified by observing that the fin seemed significantly stiffer when executing the yaw turn than during steady swimming. A second set of more flexible fin rays, with EI values $1000 \times$ those of the biological fin rays, was built for a second set of tests.

\section{Bio-robotic fin experimentation}

The manoeuvring fin was evaluated in order to assess its ability to produce motions, forces and flows similar to those made by the biological fin during the yaw turn. Experiments were also run to determine if the design retained the ability to create steady swimming forces and flows. Experimentation was conducted in two stages. Preliminary tests were conducted in a tank of static water and used to determine qualitatively if the fin was able to create movements and flows that were like those of the biological fin. The second stage of experimentation consisted of tests conducted in a flow tank during which the forces and flows produced by the fin were measured. During these tests, a fin made using fin rays with flexural rigidities scaled to $2000 \times$ the biological fin rays was tested in still water. Motions were captured using high speed video, and forces were measured. A second set of trials was conducted with fin rays $1000 \times$ the biological fin rays and which was operated in static water and in a flow. In addition to recording motions and forces as before, digital particle image velocimetry (Willert and Gharib 1991) was used to image the hydrodynamics of the flow.
The fin and motor assembly were suspended from a carriage that rested upon air bearings (New Way S301301, New Way Air Bearings, Aston, PA), which were mounted on the top of a flow tank (Figure 8) (Tangorra et al. 2008). The carriage could translate fore and aft and laterally or could be fixed against two s-beam load cells (Futek L2357, Futek Advanced Sensor technology, Irvine, CA, US) so that thrust and lateral forces could be measured.

Along with forces, fin motions and the resulting flows were measured. High speed (500 fps), high definition video (1024 × 1024) (Photron 1024PCI, Photron USA, Inc., San Diego, CA, US) was taken of the fin from both the lateral and ventral views. Hydrodynamics were visually identified by introducing a small amount of coloured dye into the water as the fin was actuated. Flows were then quantified using DPIV (Willert and Gharib, 1991; Drucker and Lauder, 1999) when the fin was operated in a flow.

Two sets of trials were run during which the fin motions, flows, and thrust/drag and lateral forces (contra-lateral towards the side opposite the fin, ipsi-lateral - towards the side of the fin) were measured. Trials were separated by several minutes to ensure that flow had come to a rest after the previous trial. The first set used a fin with $2000 \times$ fin rays and was operated with zero flow. The fin was actuated to complete the manoeuvring motion in 3.5, 1.8, 1.2 and $1.0 \mathrm{~s}$ cycle times, which spanned a range of fin velocities used on previous fins. A steady swimming stoke was executed in periods of 3.0, 1.5 and $1.0 \mathrm{~s}$. Steady swimming motions were executed to determine if the fin was capable of creating forces similar to those made by a fin developed specifically for that stroke (Tangorra et al. 2008). To do so, the fin rays on the manoeuvring fin were driven using trajectories developed for the steady swimming fin, despite the fin base having structural differences that prevented the fin rays from precisely following the steady swimming paths. The greater amount of curvature between the fin rays' centres of rotation on the manoeuvring fin meant that it would not be able to create the same amount of cupping seen on the steady swimming fin. For the second set of trials, the fin was given $1000 \times$ fin rays and operated in a flow of $90 \mathrm{~mm} / \mathrm{s}-$ a value that matched a speed used in experiments on other fins. The same flow speed was used across all trials. The yaw turns were activated in $2.3,1.3$ and $1.0 \mathrm{~s}$ and the steady swimming motion with $2.0,1.0$ and $0.7 \mathrm{~s}$ periods $(0.5,1.0$ and $1.4 \mathrm{~Hz}$ ).

Force data were collected at $200 \mathrm{~Hz}$ (National Instruments 6035, National Instruments Corporation, Austin, TX, US). Representative results for the force produced by a single stroke cycle (outstroke, instroke) were made by averaging the force from three independent cycles and low pass filtering the averaged result at $2 \mathrm{~Hz}$ and $4 \mathrm{~Hz}$ for the two set of trials, respectively. The low pass filter was designed using the Kaiser window method to have a passband frequency of $2 \mathrm{~Hz}$ and a stopband frequency of $4 \mathrm{~Hz}$ for the stiffer fin and a passband frequency of $4 \mathrm{~Hz}$ and a stopband frequency 
Major axis rotation of fin ray 4
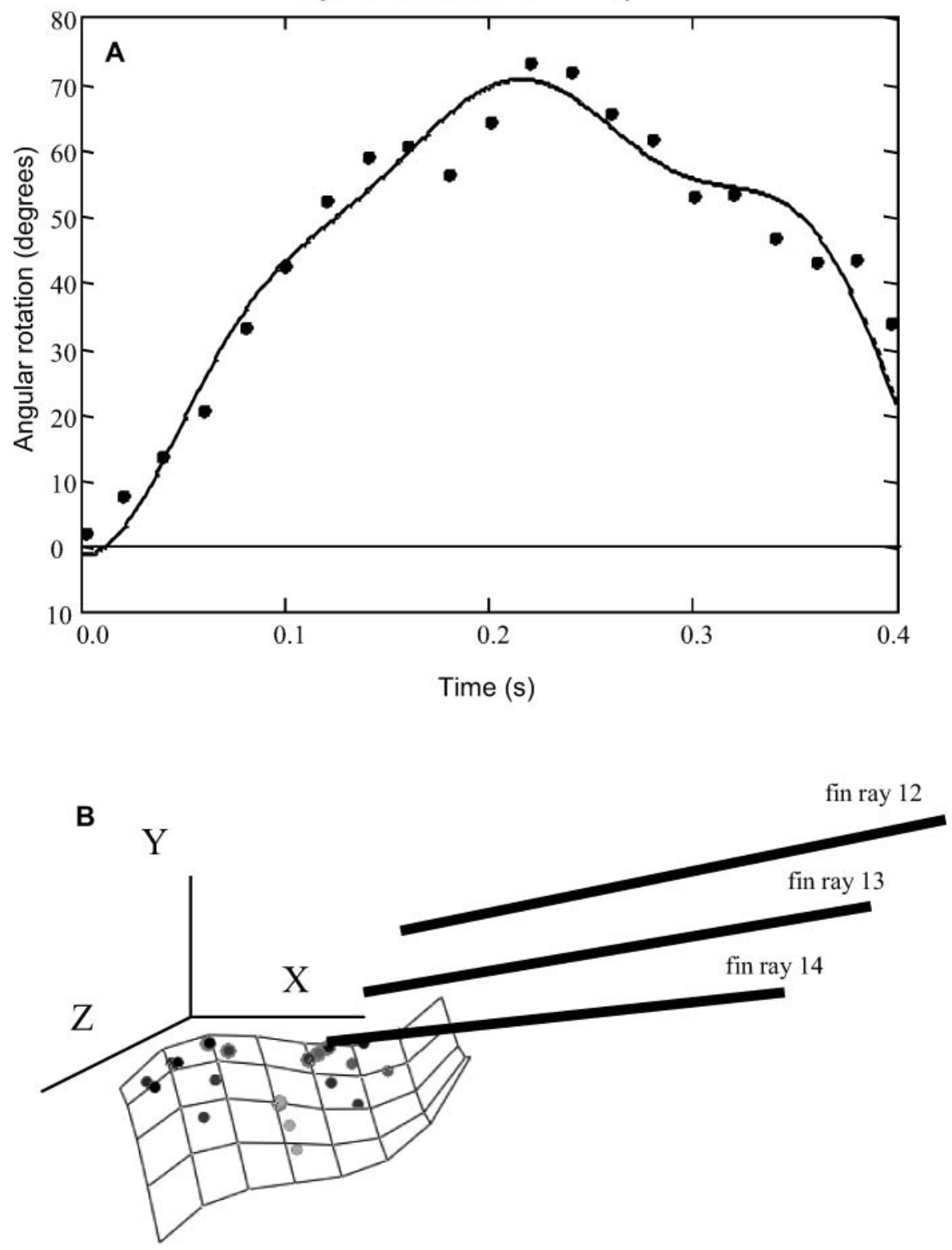

Figure 6. (A) Fitting a trajectory to fin ray 4's main plane of rotation. Each point represents the angle made by a triplet's orientation to a reference at that point in time. (B) Fin ray 14's base points through selected time steps during yaw turn. The curved, fitted plane shows the combined sweep and lateral motions of the fin ray's base points. The fin ray starts in the XY plane and moves into the $+Z$.

of $6 \mathrm{~Hz}$ for the flexible fin, each with a peak error of $10^{-3}$ (Oppenheim et al. 1999). Data were filtered to generate force curves representative of the fin's overall performance rather than highlight smaller oscillations in forces. These oscillations occurred mainly in the thrust measurements, but for consistency the low pass filter was applied to both thrust and lateral measurements. The effectiveness of the fin at producing thrust was evaluated by calculating the impulse imparted to the water by the fin in the thrust and the lateral directions. The impulse, which equals the change in momentum of the water, was approximated by numerically integrating the area under the force curves over a given period using a trapezoidal method (Press et al. 2007).

\section{Results}

\subsection{Fin motions}

The dynamic movement of the stiff $(2000 \times)$ and flexible $(1000 \times)$ robotic fins were good approximations of the biological fin's motion during the yaw turn manoeuvre (Figure 9). The creation of motions like the biological fin's 

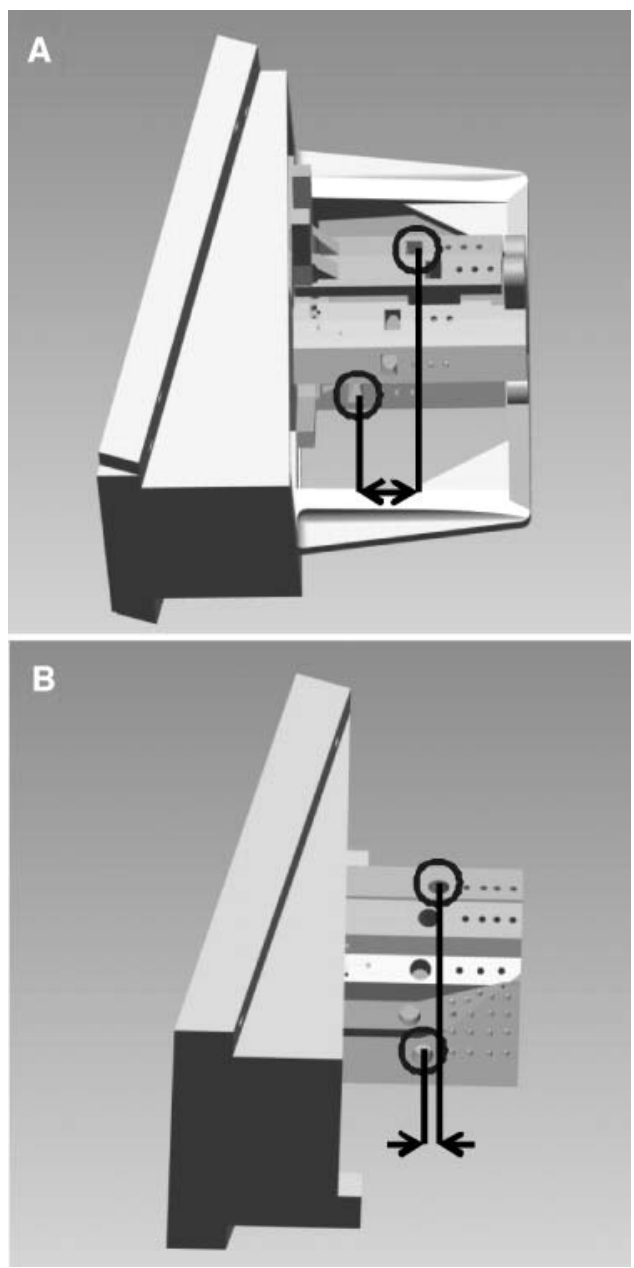

Figure 7. CAD models of fin bases for the (A) the manoeuvring fin, and (B) the steady swimming fin. The distance between the attachment points for the leading edge fin rays (circled) is shown.

is not unto itself critical, but it is an important factor in generating $3 \mathrm{D}$ forces and flows that vary in direction and magnitude throughout the fin beat like the biological forces and flows. Qualitative comparisons of fin motions were conducted by making a frame by frame comparison of the high definition, high speed video of the robotic and biological fins. Due to differences in the size of the biological and robotic fins, the numbers of fin rays, and movement of webbing etc., quantitative evaluations of the difference in fin motions (e.g. MSE) did not yield a more useful description than the qualitative comparison. The shape of the webbing and deflection of the fin rays compared favourably with the fish's fin at key times. The initial sweep of the fin's ventral half to start the outstroke created a smooth curve in the webbing from the midline to the bottom (Figure 9A). Shortly after, the dorsal half swept forward, during which significant bending of the fin occurred along the longest fin rays (Figure 9A). The ventral fin rays of the biological fin swept further away from the fish body than the ventral rays on the

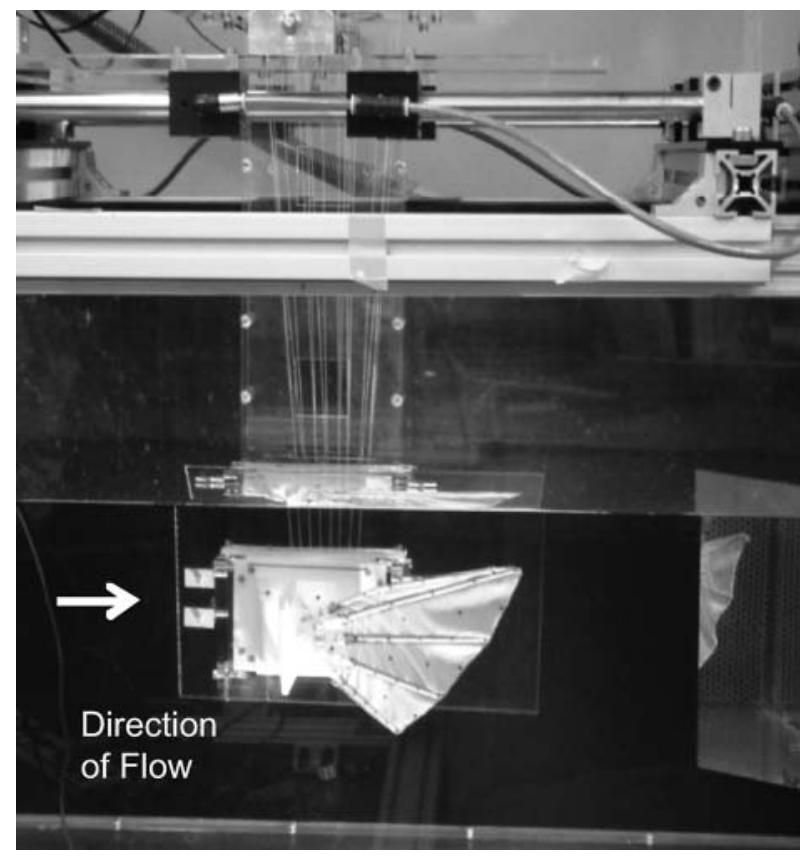

Figure 8. Experimental set-up. The fin mount is suspended from the air bearing carriage, allowing for frictionless movement and accurate force measurement. The mirror captures the lateral view of the fin during its motion. Thrust forces are directed to the left and right of the figure, and lateral forces are directed into the page.

bio-robotic fin did from the body plate (Y3 in Figure 3). This is due to the mechanical limitations of the bio-robotic fin; the angular displacement of the fin rays in the sweep direction was limited in order to simplify the construction of the fin base. On the instroke, the ventral half of the fin realigned with the dorsal half and the rays on the upper and lower edges led the fin's motion back towards the body (Figure 9B). Less deflection was seen in the bio-robotic fin rays, at this point, than was seen in the biological fin (Figure 9C).

The more flexible fin $(1000 \times)$ moved through the water in a manner very similar to that of the stiffer $(2000 \times)$ fin but with a greater amount of fin ray bending. The additional bending of the $1000 \times$ fin rays bent was visually more similar to the biological fin than the bending of the $2000 \times$ fin.

\subsection{Forces}

\subsubsection{The yaw turn}

In all but a few experimental trials, the robotic fins generated forces that were structured the same as those predicted for the biological fin: a drag and strong contra-lateral force during the fin's outstroke and smaller levels of thrust and ipsi-lateral forces during the instroke. Only at the slowest flapping speeds was there much difference between the numerical and experimental force profiles. In these cases, the stiffest fin produced drag forces during the outstroke 

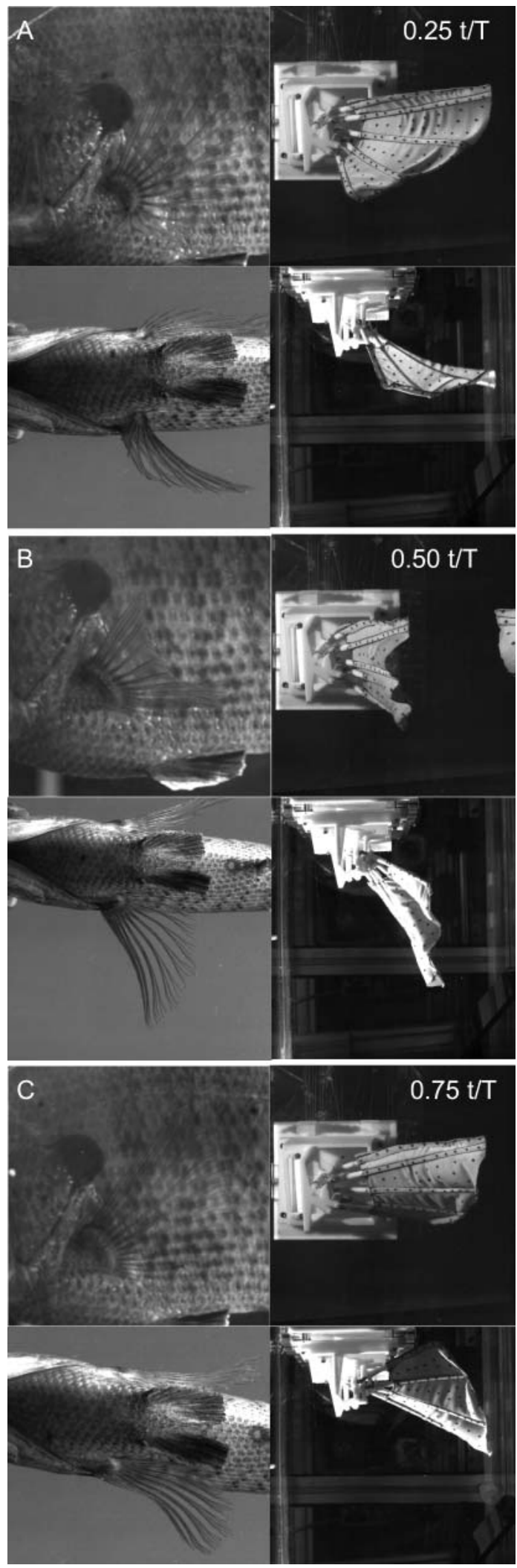

Figure 9. Lateral and ventral views of the biological and biorobotic fins at key times during the yaw turn. The bio-robotic fin is fitted with $2000 \times$ fin rays. and thrust and ipsi-lateral forces during the instroke, which were larger in magnitude than predicted forces.

The timing and magnitudes of the peak experimental and numerical (CFD) forces matched well (Figure 10). During the fin's outstroke, the peak magnitude of contra-lateral force ranged from 2.0 times as large as the drag force at the slowest fin speed (an activation time of $3.5 \mathrm{~s}$ ) to 3.4 times as large as the drag force at the fastest speed (1.0 $\mathrm{s}$ activation time). This compared well to the numerical predictions where the ratio of peak contra-lateral force to peak drag force was always approximately 2.8 . The peak contra-lateral forces developed by the robotic fin and in the simulation occurred approximately $20 \%$ into the full stroke $(t / T=0.2)$. Peak drag forces occurred between $t / T=0.3$ and 0.4 in the simulation and at approximately $t / T=0.25$ to 0.3 experimentally. These force peaks occurred later into the full stroke as the fin's flapping speed increased. The forces created during the fin's instroke of the bio-robotic fin and of the simulation were also comparable. Small amounts of thrust and ipsi-lateral force were developed, slightly more of which were seen in the forces produced by the bio-robotic fin than were predicted by the simulation.

The 2D reconstruction of the experimental forces, lateral and thrust, illustrates how the force vector would drive the fish body during the manoeuvre (Figure 11). The resultant force on the outstroke is directed in a way that would manoeuvre the fish back and away from an obstacle and is larger in magnitude than the force on the instroke. The combined forces during the instroke are in the opposite direction: thrust and ipsi-lateral force.

\subsubsection{Fin Ray Stiffness. Peak magnitudes of all forces} created by the stiffer fin were greater than those created by the more flexible fin. On the outstroke, the ratio of the contra-lateral's peak magnitude to the peaks of other forces was higher than what was predicted by CFD, although the relative sizes of the other force peaks remained consistent save for the drag force at the slowest activation speed. At all other speeds, the outstroke was dominated by the contralateral force, which was much larger than the drag force produced at the same time. The amount of drag created by the stiffer fin on the outstroke was slightly larger relative to the contra-lateral force than it was on the flexible fin. It is believed that this occurred because the stiffer fins did not bend as much and caused the fin to present a larger area to the incoming flow. For the same reason, the stiffer fin rays were largely responsible for creating higher peak forces on the instroke than were seen on either the flexible fin or CFD.

\subsubsection{Flapping Speed (Execution period). In general,} the magnitude of the peak forces during the outstroke and instroke increased as the period over which the fin executed its manoeuvre decreased. The mean forces did not follow the same trend and in some cases levelled off or decreased as flapping speed was increased. 

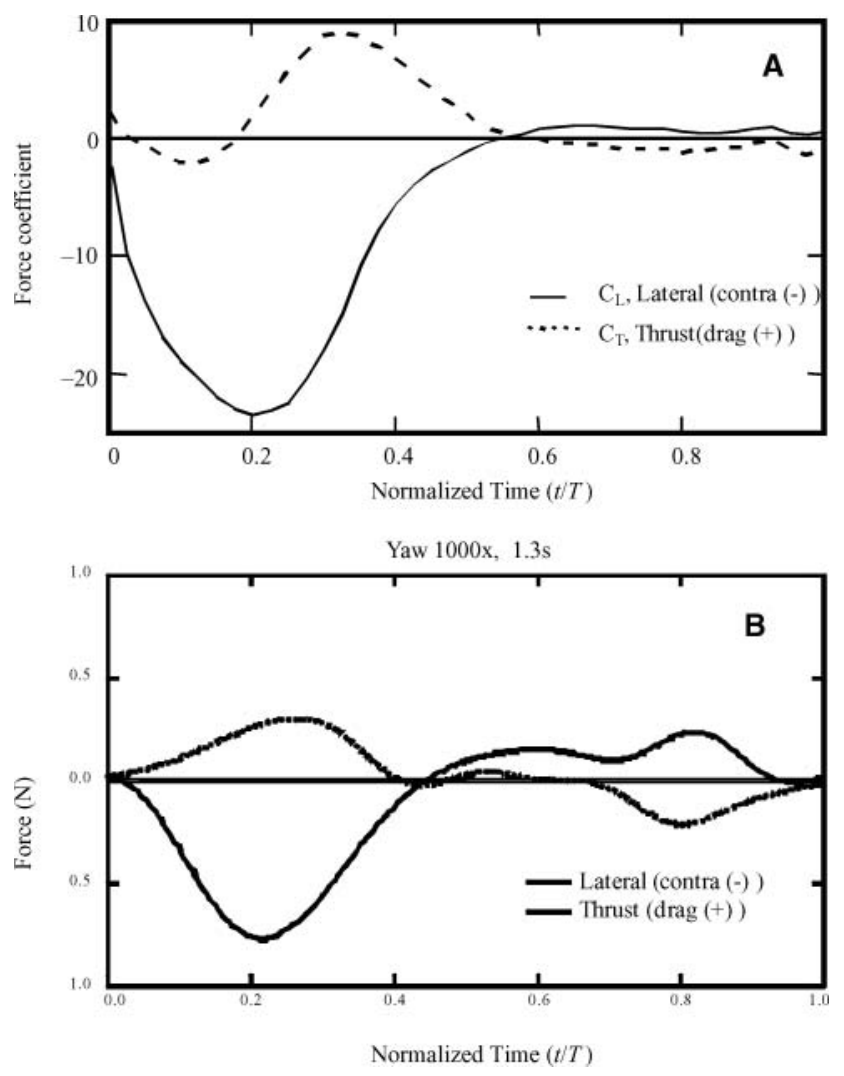

Figure 10. (A) CFD simulation of the biological fin. Traces shown are force coefficients. Figure courtesy of Rajat Mittal. (B) Forces produced by the bio-robotic fin performing the yaw turn. Drag is shown as positive for consistency with frame A.

Although the fin generated the largest mean contralateral force when flapped at its fasted speed, it produced the second smallest and smallest amounts of mean drag force on the stiff (Figure 12C) and flexible fins' (Figure 12A)

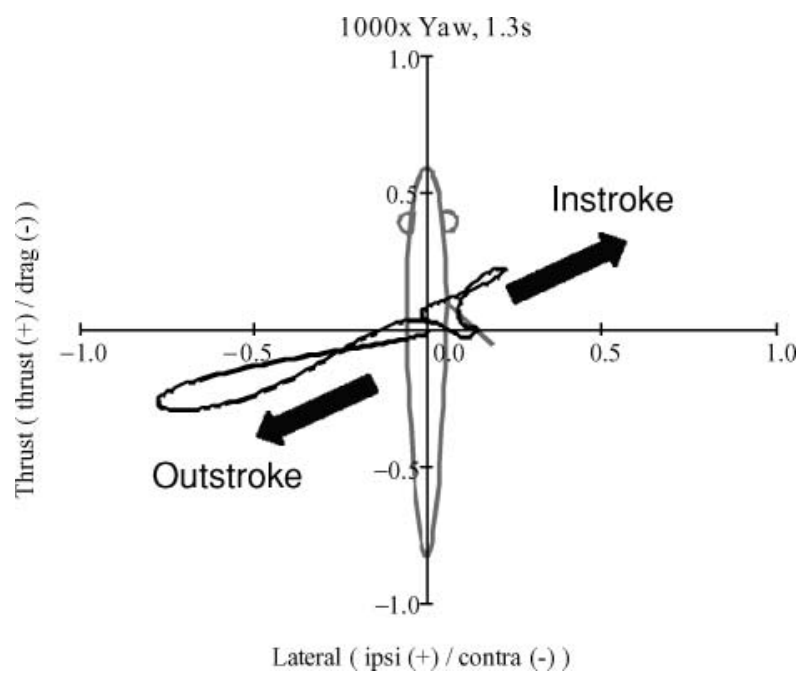

Figure 11. 2D forces in the plane of the fish. The outstroke produces a resultant force oriented in the lower left quadrant: drag and contra-lateral force. An approximate outline of the fish body and fin are shown in grey. outstrokes, respectively. The mean drag force decreased with speed for the flexible fin because the peak force remained relatively unchanged as the duration of the outstroke lessened. In contrast, the mean drag force saw relatively little change as speed was increased on the stiffer fin, since the outstroke's shortening time was offset by the increasing peak force. Mean contra-lateral force increased with speed for both fins, though did so at a much faster rate on the stiffer fin (Figure 12D), since the peak force on the flexible fin (Figure 12B) increased by smaller amounts as speed increased.

Drag forces produced on the outstroke by each fin followed different trends as speed was increased, but a common trend was shared by the fins' contra-lateral forces. As speed was increased, greater increases in peak drag force were seen on the outstroke of the stiffer fin than of the more flexible fin, where peak drag values reached their maximum at the middle operating speed. Contra-lateral force on the outstroke increased in peak magnitude consistently with speed on both fins. On the flexible fin, the mean contralateral force plateaued before the fastest speed was reached.

On the instroke, peak and mean forces increased with speed on the more flexible fin. For the stiffer fin, all forces reached a maximum at the second fastest speed (a cycle time of $1.2 \mathrm{~s}$ ). 
A

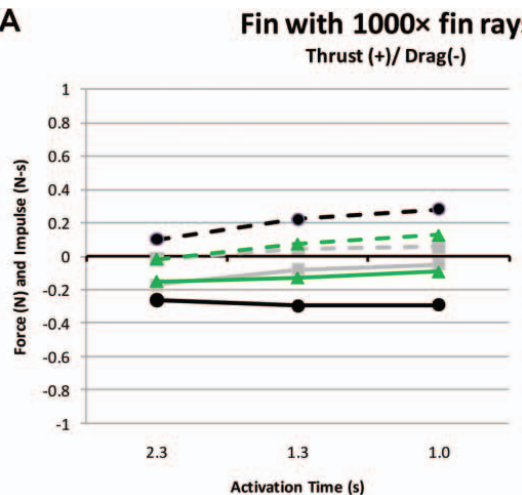

C
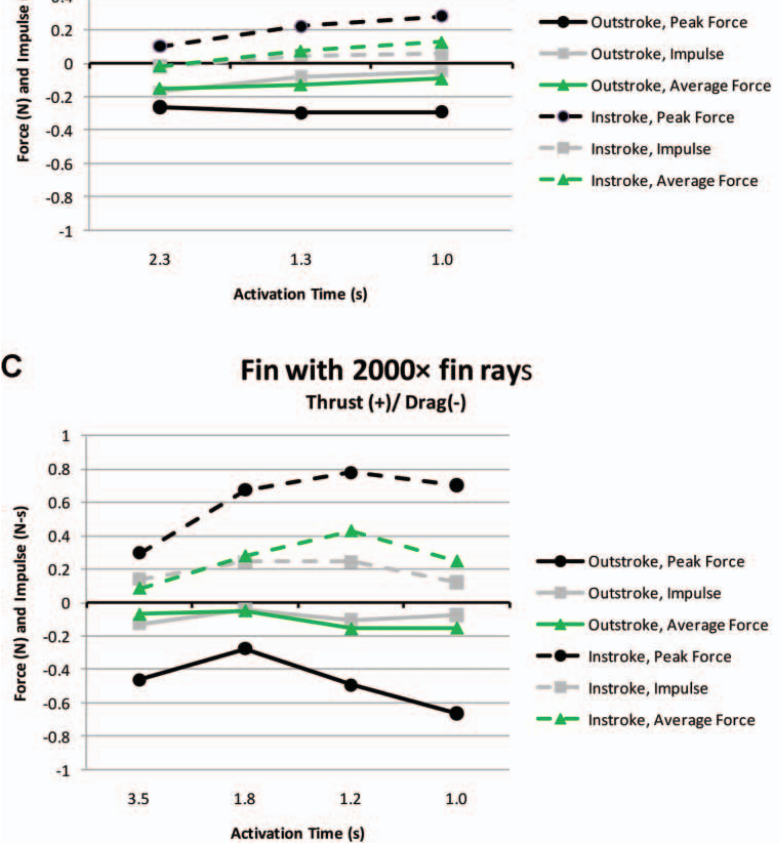

B

D

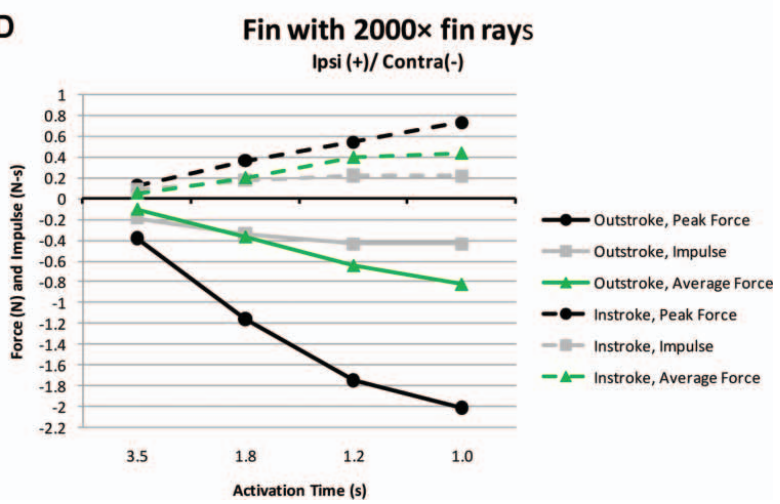

Figure 12. Peak force, impulse, and average force related to thrust and lateral forces for the outstroke and instroke of each fin. The fin with $2000 \times$ fin rays was operated in still water $(C$ and D), and the fin with $1000 \times$ was operated in a flow $(A$ and $B)$.

\subsubsection{Steady swimming}

When the fin with $2000 \times$ fin rays executed steady swimming trajectories, it produced drag and a contra-lateral force during the outstroke and thrust and an ipsi-lateral force during the instroke (Figure 13A). The drag force was of a similar magnitude to the thrust force, and the contra-lateral force was of a similar magnitude to the ipsi-lateral force. These forces were consistent with the forces created by robotic fins designed specifically for steady swimming and with fins rays of $2000 \times$ stiffness (Tangorra et at. 2007). Similarly, when the fin with $1000 \times$ fin rays executed steady swimming trajectories, a peak of drag was created on the instroke and a larger peak of thrust on the instroke. While the stiffness value of $1000 \times$ was shown to create peaks of thrust on the outstroke and instroke of the steady swimming fin (Tangorra et al. 2008), the manoeuvring fin still created drag on the outstroke, though the ratio of the peak drag force to the peak thrust force produced on the instroke was less on the more flexible fin.

Although qualitatively similar forces produced during the manoeuvring motion, the timing and magnitude of the forces were different and thus created a very different vector profile in the 2D thrust-lateral force plane (Figure 13B). The outstroke saw the production of contra-lateral and drag force, as in the manoeuvre. The forces on the instroke, ipsi- lateral and thrust, were of magnitudes quite similar to the contra-lateral and drag forces created on the outstroke. As a consequence, the resultant force vector from the instroke is close to being opposite in direction and magnitude from that of the outstroke - a stroke that would move the fish body in a very different manner than during the manoeuvre. During the yaw turn, forces on the outstroke were larger than those on the instroke, and the ratios between forces (contra-lateral to drag and ipsi-lateral to thrust) also differed.

\subsection{Flows}

As the fin moved away from the plate, a vortex formed on the tip of the upper leading edge (Figures 14C, 15). A jet of fluid was formed behind the vortex and directed away from and normal to the fin. As the fin returned to the plate on the instroke, the jet produced on the outstroke continued away from the fin, and the vortex was shed into the flow.

Both the vortex and jet were visible by using DPIV (Gericke 2009) and by observing the motion of a small volume of dye as the fin was actuated in a tank of static water. In DPIV the vortex was clearly visible as a quickly rotating mass of water past the tip of the fin. The jet is visible as the mass of water that is being accelerated between the fin and vortex directed away from the fin. The timing and 

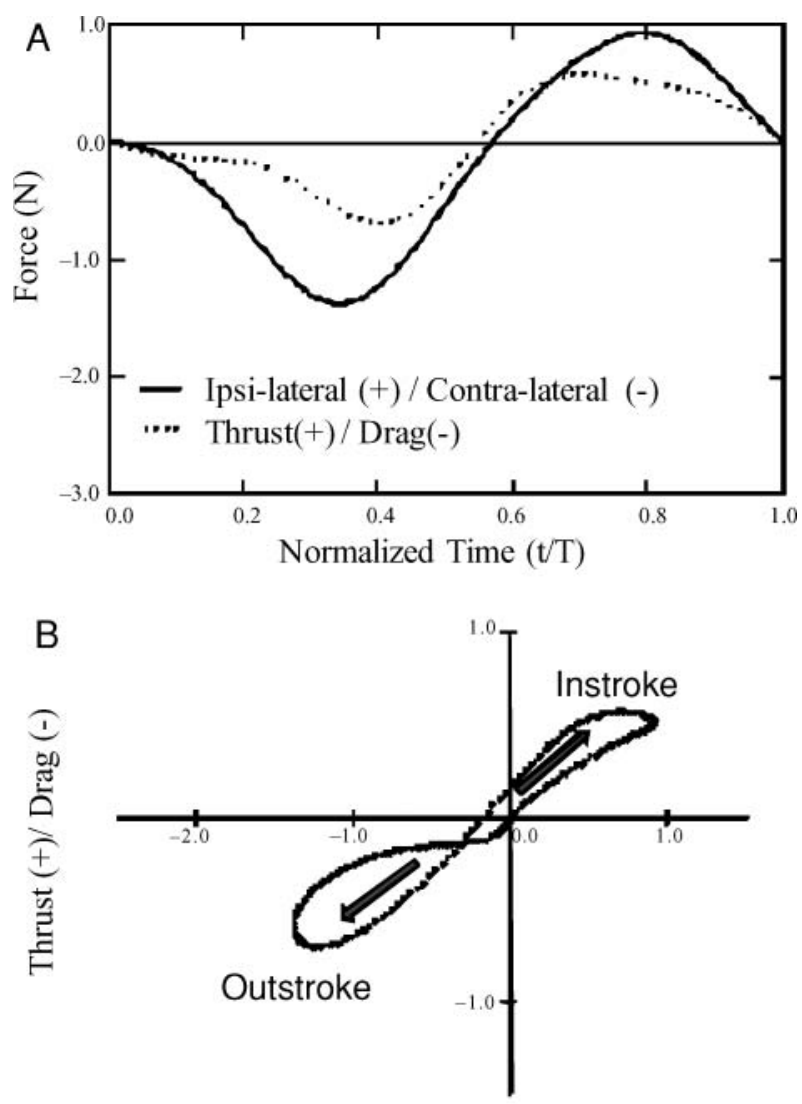

Ipsi-lateral (+) / Contra-lateral (-)

Figure 13. (A) Lateral (solid) and thrust (dotted) steady swimming forces produced during a $1.0 \mathrm{~s}$ period with $2000 \times$ fin rays. Traces are averages of several fin beats. (B) 2D force trace of total force produced by the fin during the fastest steady swimming beat. The front of the fish is along the positive y-axis.

orientation of the jet were predicted by CFD, and the reaction force to the acceleration of this mass of water away from the fin is the contra-lateral force developed on the outstroke, responsible for pushing the fish body in the opposite direction (Ramakrishnan et al. 2008). The drag force developed at this time is also visible in the DPIV, as the collection of flow vectors around the fin oriented upstream.

\section{Discussion}

\subsection{Kinematics of the yaw turn manoeuvre}

The yaw turn manoeuvre demonstrates the remarkable control that the sunfish has over its pectoral fins. The manoeuvre - during which the fish slowed its velocity, moved downward, turned away from an obstacle and then reoriented into the flow - was executed using a single fin beat. This contrasts with the cyclic motions that are used in steady swimming and which are often modelled by fin based propulsors. During this fin beat, the kinematics and stiffness of the fin were modulated so that an appropriate 3D force was cre-
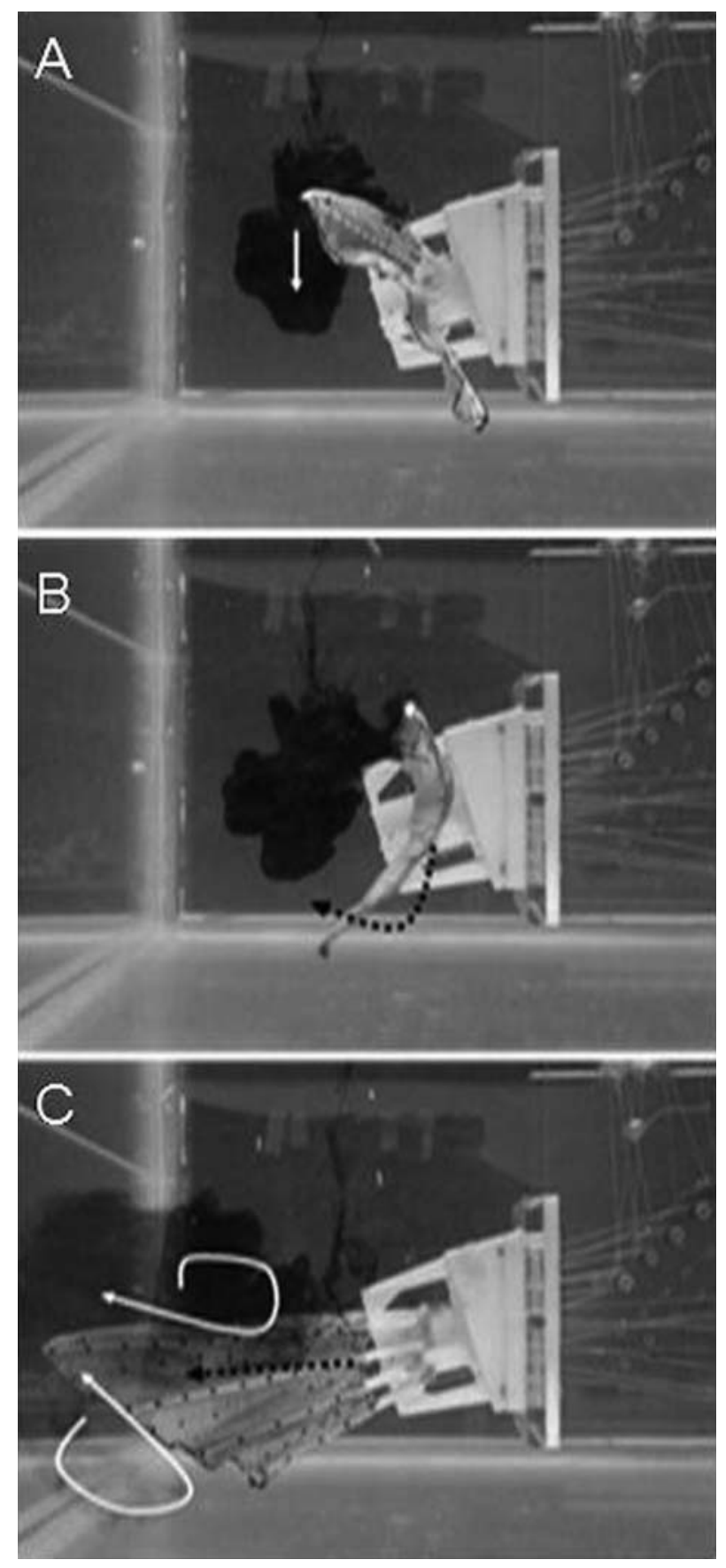

Figure 14. Posterior view of the fin executing the yaw turn. The introduction of dye enabled the identification of the resulting hydrodynamics. Solid lines indicate the movement of the dye, and dotted lines, the overall motions of the fin at select times throughout the manoeuvre.

ated to control the fish body throughout the turn. The ability to do this requires great control over the many active and passive degrees of freedom in the fin and suggests that the fins are controlled via a closed loop sensorimotor system. This may be done through sensors intrinsic to the fin and/or sensory systems extrinsic to the fin (e.g., the lateral line). 


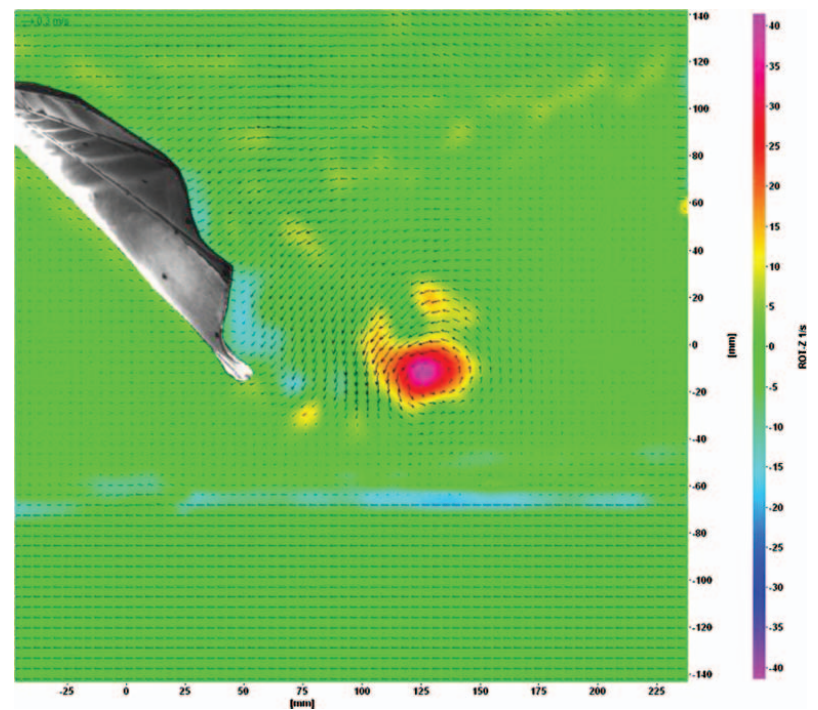

Figure 15. DPIV analysis of a $1.3 \mathrm{~s}$ yaw turn. The fin (white) is rotating clockwise from the upper left corner as it sweeps upstream during the outstroke (flow moves from left to right). Arrows represent velocity vectors of the reflective particles.

Although much is known about the sensorimotor control of terrestrial vertebrates, little information is available on the sensorimotor control systems of fish.

\subsection{Bio-robotic modelling}

The bio-robotic fin is a greatly simplified model of a complex biological system but captured the characteristics essential to create forces and flows like the biological fin and had sufficient active and passive degrees of freedom to generate a range of biologically and non-biologically derived motions. This was due primarily to successful modelling of the kinematics and physical properties of the fin's rays. In addition to obvious differences between biological and human engineered systems, crucial simplifications included modelling the 14 biological fin rays using 5 robotic rays, treating trajectories for the fin rays as 2-DOF rotations and modelling passive fin ray flexural rigidities using tapered rays with a single scaling factor. As for the robotic fins that were developed for the steady swimming fins (Tangorra et al. 2006, 2008), the five fin rays $(1,4,7,10,14)$ which best bounded the complex shape of the biological fin as it moved through the fin beat were selected. These fin rays provided control over the upper and lower leading edges and the upper and lower halves of the fin. The use of more rays would have provided additional control over the fin's movement and would have decreased the area of webbing that did not have fin ray support but would have done so at the expense of complexity. In robotic fins that are being designed to capture manoeuvring and steady swimming, each fin ray requires up to three actuators. It was therefore important to identify the minimum number of fin rays and actuators that was sufficient to execute the movements used in the turn manoeuvre. Reducing complexity extended, also, to the fin ray trajectories. Although the biological fin rays translated as well as rotated, the robotic fin rays were only made to rotate - fins rays 1,10 and 14 had two degrees of rotational freedom, while fin rays 4 and 7 had one. The translation of the fin ray bases was small during the fin beat, and so it was deemed sufficient to give the fin ray bases a stationary point about which to rotate. The success of the fins in creating forces like those predicted using CFD supports this simplification. This likely holds, however, only for a robotic fin designed specifically for the turn manoeuvre. Since the relative positioning of the fin ray bases influences the shape of the fin webbing as the fin rays are swept forward, the translation of the fin ray bases is important when adjusting the shape of the base so that the fin can execute different types of fin beats.

Just as in steady swimming (Tangorra et al. 2008), the proper scaling of fin ray stiffness during the manoeuvre impacted the ability of the fin to produce forces that were consistent with CFD predictions. Initially, the stiffness scaling for the fin rays was chosen qualitatively; the biological fin appeared stiffer during the yaw turn than during steady swimming, and so the robotic fin rays were scaled to be twice as stiff $(2000 \times)$ as fins rays used in steady swimming fins. This fin appeared too stiff, and created forces that were somewhat inconsistent with CFD predictions: the drag created during the outstroke was high, as were the magnitudes of the thrust and ipsi-lateral forces during the instroke. Although the directions of these force components were correct, their larger magnitudes meant that they combined to create a force vector that had the wrong magnitude and direction to manoeuvre the fish appropriately. The fin was then tested with more flexible fin rays scaled to $1000 \times$ - the stiffest value used on steady swimming fins that produced thrust during the outstroke. With these more flexible fin rays, the manoeuvring fin produced forces and flows more consistent with CFD; the relative sizes of peak forces, the timing at which they occurred and the resulting hydrodynamics all matched predictions well. While it is possible that the improvement from the stiffer fins rays to the more flexible ones in matching CFD was impacted by the addition of flow, it likely was not as significant a factor as fin ray stiffness itself. It can be assumed that significantly more flexible fin rays would not have been able to produce the strong lateral jet seen on the outstroke, as too much of the fin would have bent away from the incoming flow. It is apparent that, just as in steady swimming, fin ray stiffness for the yaw turn must be properly tuned and adjusted actively so that the fin can be adjusted for different flow conditions and fin beats.

\subsubsection{Steady swimming forces}

The inability of the fin with $1000 \times$ stiffness to create thrust during the outstroke of the steady swimming movement 
strongly supports the assertion that superficial modelling of a biological system is often insufficient if the engineered system is to exploit the physical phenomena that give the biological system its special abilities. Although this robotic fin was designed specifically to execute a yaw turn manoeuvre, it shared many of the same physical characteristics as the robotic fins made for steady swimming, and the two degrees of rotational freedom that its fin rays had made it possible to approximate the trajectories of the steady swimming fin. Tests conducted with the stiffer $2000 \times$ fin produced forces that were similar to those produced by fins designed specifically for steady swimming and with the similarly stiff fin rays (i.e., drag and lift on the outstroke, thrust and downward lift on the instroke) (Tangorra et al. 2007). However, when fin ray stiffness was reduced to $1000 \times$, the fin no longer produced forces like a steady swimming fin of similar stiffness. At $1000 \times$ and lower, the steady swimming fins produced thrust during the outstroke, just as the biological fin (Tangorra et al. 2008), whereas the fin designed for the turn manoeuvre continued to produce drag during the outstroke. We believe that this was due to the manoeuvring fin not cupping sufficiently about its spanwise axis as it was swept forward. The fin rays could be driven with velocities that were correct for steady swimming and had the stiffness appropriate for a good dynamic interaction with the water. However, the most ventral rays did not curl towards the centre of the fin as they were swept forward as they do in the steady swimming fin. The was due to the rotational point of the ventral leading edge of the yaw turn fin being much closer to the body plate than in the steady swimming (Figure 7). This caused the bases of the fin rays to form a much more gradual arc than in the steady swimming fin, and as a result, the manoeuvring fin did not cup strongly about its spanwise axis. Thus, for a bio-robotic fin to create the forces for a yaw turn manoeuvre and steady swimming well, active control over the position of the fin rays within the base in addition to active control over the fin rays, velocity profile and mechanical stiffness would be required.

\section{Conclusions}

The careful analysis and adoption of the anatomy, kinematics and mechanical properties of the sunfish pectoral fin resulted in a bio-robotic fin that was able to perform like a sunfish fin during a yaw turn manoeuvre. The complex 3D shapes produced by the fin approximated those of the biological fin well, and the forces and flows closely matched predictions made using numerical models of the biological fin (Mittal et al. 2006; Ramakrishnan et al. 2008).

A series of simplifications were made when modelling the biological system that simplified it enough for robotic implementation without losing any of the aspects integral to creating the yaw turn. Five of the biological fin's fourteen fin rays were carefully chosen for use on the robotic fin. The stiffness of the fin rays was controlled with a scaling law. It was found to be sufficient to model that complex translations and rotations of the biological fin rays as 1- and 2-DOF rotations about single rotations points. Base curvature was also found to be integral to producing proper kinematics. Of particular importance was that both the kinematics and mechanical properties of the fin rays had to be altered to produce the forces appropriate for moving the fish body through the turn in a single fin beat. While it is clear that fin ray stiffness must be appropriately modelled for each stroke, a stiffness value of $1000 \times$ may be a good compromise; using this stiffness, the manoeuvring fin created forces that very closely matched those predicted by CFD for the yaw turn, and a steady swimming fin was able to create a fin beat with two thrust peaks. For a fin to be truly multi-functional, active control of base curvature, fin ray motions and fin ray stiffness are thus required if bio-robotic, fin based propulsors are to be expected to have performance on par with biological fins.

The active degrees of freedom that were employed to execute the yaw turn movements made it possible for the fin to create a range of non-biologically derived motions. Although the fin was able to move the fin rays through trajectories that were similar to those used in steady swimming, the fin did not produce thrust during the steady swimming outstroke, as does the biological fish. This was due to the fin rays of the yaw turn fin being positioned in the fin base differently from that in the steady swimming fin and supports the need for careful modelling of the biological system during the specific operating condition being addressed. It also suggests that additional active degrees of freedom should be employed to actuate the curvature of the fin base and the positioning of the fin rays within the base so that the motion for steady swimming and turn manoeuvres can be created well by the same fin.

The overall result of this research was a bio-robotic fin that was capable of creating the impulsive forces - manoeuvring forces, which were vectored through $3 \mathrm{D}$ in a single fin beat - used by the fish during a turning manoeuvre. It has laid the groundwork for a multi-functional, bio-robotic fin based propulsor, which could ultimately grant man-made underwater vehicles fish-like agility. This fin is one element in the development of a bio-robotic pectoral fin capable of producing the effects of the yaw turn, steady swimming and hovering.

\section{Acknowledgements}

This work was funded in part by ONR MURI N000140310897. Jonah Gottlieb acknowledges the contributions of a GAANN scholarship. The authors would like to thank Lauren Gibilisco for her work digitising the $3 \mathrm{D}$ coordinates of fin ray movements.

\section{References}

Alben S, Madden PG, Lauder GV. 2006. The mechanics of active fin-shape control in ray-finned fishes. J. Royal Society Interface. 4(13):243-256. 
Anderson JM, Chhabra NK. 2002. Maneuvering and stability performance of a robotic tuna. Integ. And Comp. Biology. 2002 42(1):118-126.

Drucker EG, Jensen JS. 1996. Pectoral fin locomotion in the striped surfperch. J. Exp Biol. 199(10):22352242.

Drucker EG, Lauder GV. 1999. Locomotor Forces on a Swimming Fish: Three-Dimensional Vortex Wake Dynamics Quantified Using Digital Particle Image Velocimetry. J. Exp Biol. 202:2393-2412.

Drucker EG, Lauder GV. 2001. Wake dynamics and fluid forces of turning maneuvers in sunfish. J. Exp Biol. 204(3):431442.

Fish FE, Lauder GV, Mittal R, Techet AH, Triantafyllou MS, Walker JA, Webb PW. Conceptual design for the construction of a biorobotic AUV based on biological hydrodynamics. Proceedings of the 13th International Symposium on Unmanned Untethered Submersible Technology (UUST), Durham, N.H.

Gericke T. 2009. Fish Biorobotics: Pectoral Fin Dynamics and Biomimetics in Fishes. Master's Thesis, Bremen University of Applied Sciences, Bionics and Locomotion in Fluids.

Gottlieb JR, Tangorra JL, Gibilisco L, Lauder GV. 2008. The Development of a Flapping Fin for Maneuvering. IEEE/RAS Int. Conf. on Biomedical Robotics and Biomechatronics (BIOROB); Scottsdale, Arizona, USA.

Kato N, Furushima M. 1996. Pectoral fin model for maneuver of underwater vehicles. Proceedings of the 1996 Symposium on Autonomous Underwater Vehicle Technology, Monterey, CA.

Lauder GV, Anderson EJ, Tangorra JL, Madden PG. 2007. Fish Biorobotics: Kinematics and Hydrodynamics of SelfPropulsion. J. Exp Biol. 210:2767-2780.

Lauder GV, Drucker EG. 2004. Morphology and experimental hydrodynamics of fish fin control surfaces. J. Oceanic Eng. 29(3):566-571.

Mittal R, Dong H, Bozkurttas M, Lauder GV, Madden PG. 2006. Locomotion with Flexible Propulsors: II. Computational Modeling of Pectoral Fin Swimming in Sunfish. Bioinspiration and Biomimetics. 1(4):S35-S41.
Oppenheim AV, Schafer RW, Buck JR. 1999. Discrete-Time Signal Processing. 2nd ed. Upper Saddle River, NJ: Prentice-Hall, pp. 474-478.

Palmisano J, Ramamurti R, Lu K, Cohen J, Sandberg W, Ratna B. 2007. Design of a Biomimetic Controlled-Curvature Robotic Pectoral Fin. IEEE Intl. Conf. on Robotics and Automation (ICRA); Rome, Italy.

Press WH, Teukolsky SA, Vetterling WT, Flannery BP. 2007. Numerical Recipes: The Art of Scientific Computing. Third Edition. New York: Cambridge UP, 2007.

Ramakrishnan S, Mittal R, Lauder GV, Bozkurttas M. 2008. Analysis of Maneuvering Fish Fin Hydrodynamics Using an Immersed Boundary Method. AIAA 2008 38th Fluid Dynamics Conference and Exhibit, Seattle, Washington, USA.

Tangorra JL, Davidson SN, Hunter IW, Madden PG, Lauder GV, Dong H, Bozkurttas M, Mittal R. 2007. The Development of a Biologically Inspired Propulsor for Unmanned Underwater Vehicles. J. Oceanic Eng. 32(3):533-550.

Tangorra JL, Davidson SN, Madden PG, Lauder GV, Hunter IW. 2006. A Biorobotic Pectoral Fin for Autonomous Undersea Vehicles. IEEE Eng. In Medicine and Biology Society (EMBS); New York City.

Tangorra JL, Lauder GV, Madden PG, Mittal R, Bozkurttas M, Hunter, IW. 2008. A Biorobotic Flapping Fin for Propulsion and Maneuvering. IEEE Intl. Conf. on Robotics and Automation (ICRA); Pasadena, California, USA.

Westneat MW. 1996. Functional Morphology of Aquatic Flight in Fishes: Kinematics, Electromyography, and Mechanical Modeling of Labriform Locomotion. J. Amer. Zool. 36:582-598.

Wilga CD, Lauder GV. 1999. Locomotion in Sturgeon: Function of the Pectoral Fins. J. Exp Biol. 202(18):2413-2432.

Wilga CD, Lauder GV. 2000. Three Dimensional Kinematics and Wake Structure of the Pectoral Fins During Locomotion in Leopard Sharks Triakis Semifasciata. J. Exp Biol. 203(15):2261-2278.

Wilga CD, Luader GV. 2001. Functional Morphology of the Pectoral Fins in Bamboo Sharks, Chiloscyllium plagiosum: Benthic vs. Pelagic Station-Holding. J. Morphology. 249(3):195209.

Willert CE, Gharib M. 1991. Digital Particle Image Velocimetry. J. Exp. Fluids. 10, 181-193. 

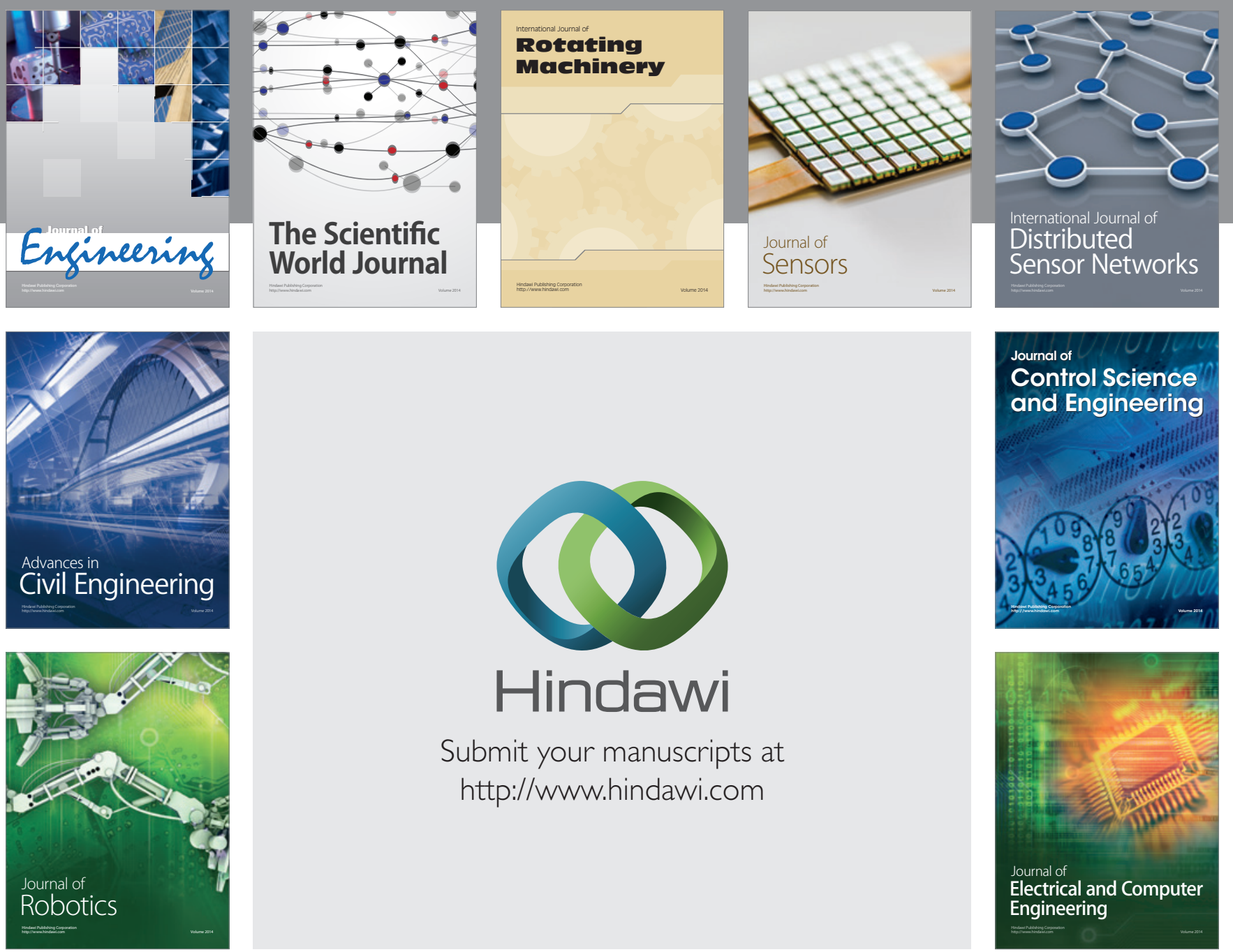

Submit your manuscripts at

http://www.hindawi.com
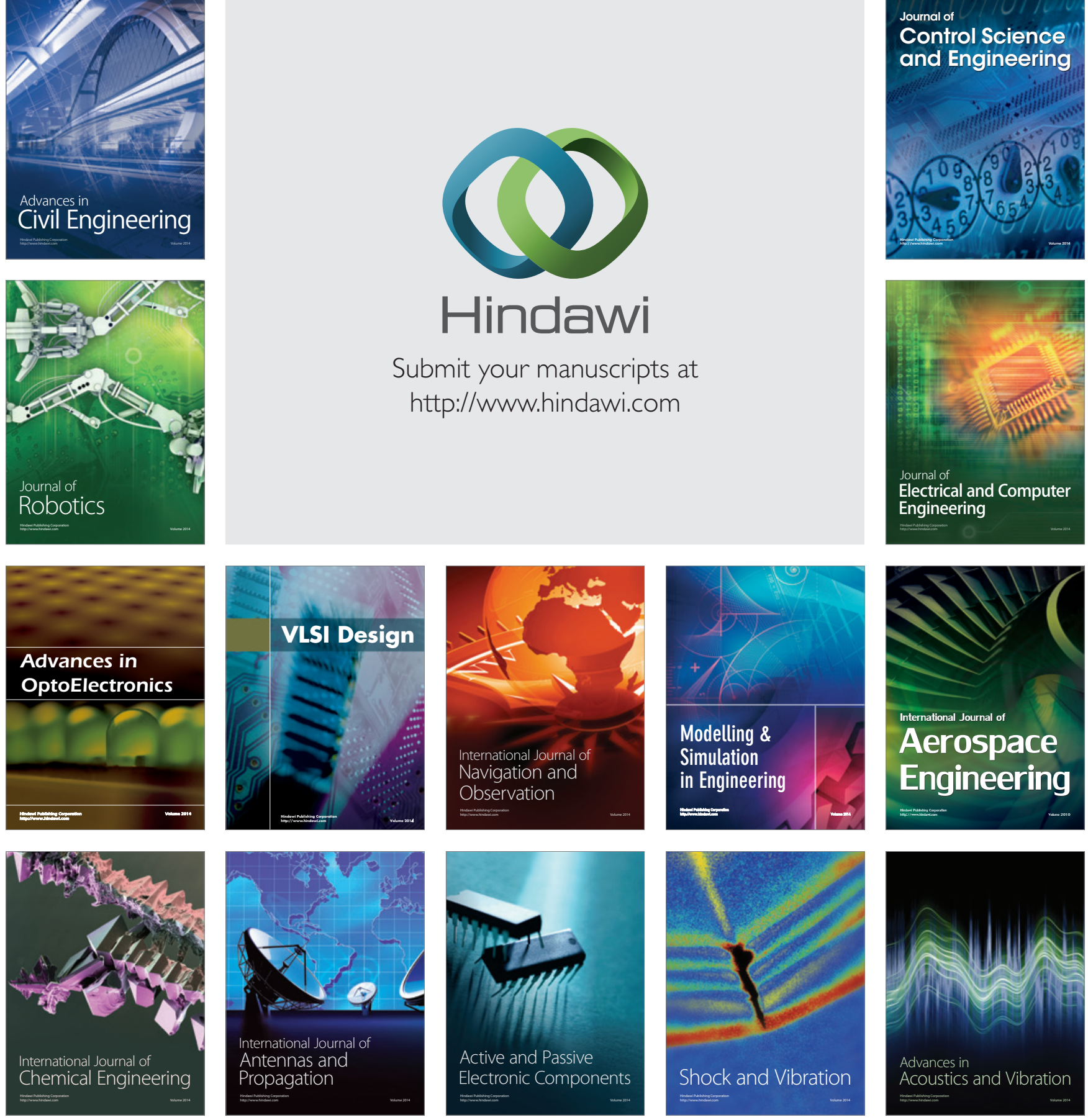